\title{
Review of: "Open Access"
}

\author{
Scott Abbott ${ }^{1}$ \\ 1 University of Technology Sydney
}

Potential competing interests: The author(s) declared that no potential competing interests exist.

Well thought out and comprehensive definition of open access. It is standing well against the test of time. 